\title{
Penile Cancer Disparities in Puerto Rican Men as compared to the United States Population
}

Vivian Colón-López, Ana P. Ortiz, Marievelisse Soto-Salgado, Mariela Torres-Cintrón; Curtis A. Pettaway, Antonio Puras-Báez; Magaly Martínez-Ferrer, Erick Suárez

Department of Health Services Administration, Graduate School of Public Health, Medical Sciences Campus, University of Puerto Rico (VCL), Comprehensive Cancer Center, University of Puerto Rico (VCL, MMF), Department of Biostatistics and Epidemiology, Graduate School of Public Health, Medical Sciences Campus, University of Puerto Rico (APO, ES), Cancer Control and Population Sciences Program, PR Comprehensive Cancer Center (APO), UPR/MDACC Partnership University of Puerto Rico Comprehensive Cancer Center (MSS), Puerto Rico Central Cancer Registry, University of Puerto Rico Comprehensive Cancer Center (MTC), MD Anderson Cancer Center, Department of Urology, Division of Surgery (CAP) and Department of Surgery - Urology Section, Medical Sciences Campus, University of Puerto Rico (APB, MMF), San Juan, Porto Rico

\section{ABSTRACT}

Purpose: This study compares incidence and mortality of penile cancer in Puerto Rico (PR) with other racial/ethnic groups in the United States (US) and evaluates the extent in which socioeconomic position index (SEP) or its components influence incidence and mortality in PR.

Materials and Methods: Age-standardized rates were calculated for incidence and mortality based on data from the PR Cancer Registry and the US National Cancer Institute's Surveillance, Epidemiology and End Results program, using the direct method.

Results: PR men had approximately 3-fold higher incidence of penile cancer as compared to non-Hispanic white (Standardized rate ratio [SRR]: 3.33; 95\%CI=2.80-3.95). A higher incidence of penile cancer was also reported in PR men as compared to non-Hispanic blacks and Hispanics men. Mortality from penile cancer was also higher for PR men as compared to all other ethnic/racial groups. PR men in the lowest SEP index had 70\% higher incidence of penile cancer as compared with those PR men in the highest SEP index. However, the association was marginally significant (SRR: 1.70; 95\%CI=0.97, 2.87). Only low educational attainment was statistically associated with higher penile cancer incidence (SRR: 2.18; 95\%CI=1.42-3.29).

Conclusions: Although penile cancer is relatively uncommon, our results support significant disparities in the incidence and mortality rates among men in PR. Low educational attainment might influence the high incidence of penile cancer among PR men. Further studies are strongly recommended to explore these disparities.
ARTICLE INFO

\section{Key words:}

Urogenital Neoplasms; Penile

Neoplasms; Epidemiology;

Minority Health; Healthcare

Disparities

Int Braz J Urol. 2012; 38: 728-38

Submitted for publication:

April 04, 2012

Accepted after revision:

August 01, 2012

\section{INTRODUCTION}

Increased insight has been gained into the pathogenesis of penile cancer, since reports indi- cated that several risk factors such as phimosis with chronic inflammation, lack of circumcision, human papillomavirus (HPV) infection, poor hygiene and smoking history are associated with pe- 
nile cancer (1). Some of these risk factors, including HPV infection, circumcision, smoking and hygiene, have been previously identified as modifiable risk factors that might lead to a reduction of penile cancer incidence and mortality (2).

The incidence of penile cancer varies enormously among different populations, being highest in developing countries (3). An evaluation of cancer registries around the world shows that although penile cancer is a rare disease with lowest rates in Israel (4), Western Europe and the United States (US) $(<1$ per 100,000) (5), incidence rates of 4.0 and 3.7 per 100,000 have been reported in Uganda and Brazil, respectively (6). In the US, although penile cancer represents less than $1 \%$ of new cancers in men, rates among Hispanics (USH) are $72 \%$ higher compared to Non-Hispanics (7). These higher estimates of penile cancer among Hispanic men correlate with the high incidence of cervical cancer among USH women in the US compared with non-Hispanic whites (NHW) (8). Penile incidence rates from a limited time period (1995-1999) also shows a high incidence rate in PR men (2.6 per 100,000) (9).

Despite the low burden of penile cancer incidence and mortality in the US, it is important to assess the recent distribution of this malignancy within specific racial/ethnic groups, particularly those of Hispanic origin. This will be important in order to identify vulnerable groups and evaluate the impact that HPV-related cancers in men might have on cervical cancer. Within the Hispanic/ Latino population, in Puerto Rico (PR), particularly, where rates of cervical and oropharyngeal cancer are higher than Non-Hispanic Whites (NHW) $(8,10)$, no studies have been performed recently to evaluate penile cancer incidence and mortality rates beyond descriptive epidemiology (11). Therefore, in this study we estimated trends in penile cancer occurrence for each racial/ethnic group from 1992 to 2004; then, we compared penile cancer incidence and mortality in men in PR with USH, NHW and Non-Hispanic Blacks (NHB), during the period 2000-2004. Finally, given the potential impact of socioeconomic factors on the burden of penile cancers in men, we evaluated the effect of socioeconomic indicators of health on penile cancer incidence and mortality among PR men.

\section{MATERIALS AND METHODS}

\section{Data sources}

Incidence statistics from PR were obtained from the Puerto Rico Central Cancer Registry (PRCCR). The PRCCR is the fourth oldest population-based cancer registry in the world collecting information on cancer in PR since 1951. The PRCCR uses the Surveillance, Epidemiology, and End Results (SEER) program and the North American Association of Central Cancer Registries (NAACCR) standards for coding data; thus, the registry is fully comparable with both SEER and NAACCR data. All penile cancer cases diagnosed since 2001 are reported using the third edition of the International Classification of Disease for Oncology (ICD-0-3) (C60.0-C60.9). Cases from 1992 to 2000 which were originally reported using previous editions of ICD-0 were converted to ICD-0-3 codes. Mortality information for PR from 1998-2004 was obtained from the PRCCR as reported by death certificates prepared by the PR Department of Health.

Penile cancer incidence statistics for USH, NHW and NHB were obtained from those released by the SEER program. The SEER program identifies Hispanic ethnicity by a combination of medical record review and matching surnames against a list of Hispanic surnames. The term Hispanic used throughout our report does not account for racial differences within the USH population. Penile cancer mortality information for USH, NHW and NHB was obtained from the SEER program as reported by the National Center for Health Statistics (NCHS). US mortality cases were obtained for all states except Connecticut, Maine, Maryland, Minnesota, New Hampshire, New York, North Dakota, Oklahoma and Vermont because of the large number of individuals with unknown origin or ethnicity ( $\geq 10.0 \%$ missing) for several years. Thus, the "Hispanic Index" as developed by the National Cancer Institute (NCI) was used to exclude states where mortality statistics for Hispanics were deemed unreliable (12).

\section{Statistical analysis}

Age-standardized rates: For each racial/ ethnic group, we applied the direct method to esti- 
mate the penile cancer age-standardized incidence and mortality (per 100,000 persons) for three time periods (1992-1996, 1997-2000, and 2001-2004) using the World Standard Population as reference. These rates were identified by ASR (World), either for incidence or mortality. The change in the ASR from the earliest and the latest studied period (1992-1996 and 2001-2004) was calculated as a percentage as follows:

$$
\text { \%change }=\frac{\text { Rate }_{2001-2004}-\text { Rate }_{1992-1996}}{\text { Rate }_{1992-1996}} * 100
$$

The significant percentage of change was determined by the construction of the 95\% confidence intervals (CI) using the formulas from the U.S. Census Bureau (http:// www.census.gov/acs/ www/Downloads/data_documentation/Accuracy/ PercChg.pdf). If zero was not included in this interval, significant changes were declared with $\mathrm{p}$ -value less than 5\%.

Group differences: To assess racial/ethnic group differences, the ASRs (World) were grouped during the study period, as follows:

$$
\operatorname{ASR}(\text { World })_{i}=\sum_{j=1}^{3} w_{j} \frac{\sum_{k=2001}^{2004} d_{i j}^{k}}{\sum_{k=2001}^{2000} n_{i j}^{k}}
$$

Then, the ratio of two standardized rates

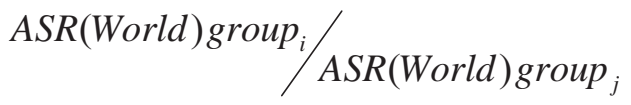

between any two groups was estimated with 95\% CIs, to assess differences in penile cancer incidence and mortality between PR group and USH, NHB and NHW groups. This ratio if referred to as the standardized rate ratio (SRR). In addition, age-specific incidence (per 100,000 persons) and mortality rates for different age groups $(<60,60-$ $70,>70$ ) was computed for the period 2001-2004. On the basis of these rates, the relative risks (RR) were estimated with 95\% CIs to determine relative differences among study groups using the Poisson regression model. The reference groups in the age-specific RR estimation were NHW, NHB, and USH.

SEP: The socioeconomic assessment was performed only for PR because the geographical level of analysis available to define the socioeconomic characteristics in PR was different from the geographical level in the US. PR penile cases diagnosed from 2001-2004 and cancer deaths from 2001-2004 were linked to the 2000 PR Census data. Briefly, principal component analysis (PCA) was used to obtain the SEP index at municipality level. The PCA transform a set of correlated variables to a new set of uncorrelated variables. Therefore, we initially considered 14 socioeconomic indicators available in the US Census 2000 for PR. Then, the most correlated socioeconomic indicators, based on the Pearson correlation index and PR data, were used for PCA. As a consequence the following eight-census based socioeconomic indicators were used for the PCA: unemployment rate, median family income, \%population living below the poverty level, \%population aged $>25$ years with less than 12 years of education, \%employed civilian population aged $>16$ years in management, professional, and related occupations (used to define white-collar occupations), \%оccupied housing units without telephone, \%population fluent in both English and Spanish, and \%occupied housing units without car (13). The first principal component was used to define the SEP due to the fact, that the rest of the principal components had a variance lower that one (criterion of Kaiser). Once the SEP was computed for every municipality, the index was categorized in five groups using quintiles as the cut-off points; such as the municipalities with the lowest socioeconomic position (highest socioeconomic deprivation) were identified by SEP1 and the municipalities with highest socioeconomic position (lowest socioeconomic deprivation) were identified by SEP5. To assess the socioeconomic effect, the two extreme SEP categories were used (SEP1 vs. SEP5) to compute the relative ratio (RR) of the ASR's of penile cancer incidence and mortality with 95\% confidence intervals. The ratio of the ASR's in these SEP categories was used to determine the socioeconomic disparity by the SEP index and the 
eight-census based socioeconomic indicators of penile cancer incidence and mortality among men in PR for the period 2000-2004.

The statistical comparisons were performed using the STATA System release 11.0 (STATA Corp, College Station, TX, USA).

\section{RESULTS}

\section{Penile Cancer Trends}

In PR, a total of 587 cases of penile cancer were diagnosed between 1992-2004. Comparing the period of 2001-04 vs. 1992-1996, penile cancer incidence decreased for all racial/ethnic groups. Increasing percent change in mortality of penile cancer was only observed among NHB and PR men. A slightly increase percent change was observed among NWH (Table-1).
Penile Cancer Rates (2000-2004)

The age-standardized incidence (per $100,000)$ of penile cancer ranged from 0.84 in NHW to 2.8 in PR. Puerto Rican men had 3 times higher incidence, than NHW (SIR: 3.33; 95\% $\mathrm{CI}=2.80$, 3.95) and NHB (SIR: 3.04; 95\%CI=2.21, 4.36). PR men had more than 2 times higher incidence than USH (SIR: 2.59; 95\%CI=1.99, 3.43) (Table-2).

The age-specific incidence increased with age among all ethnic/racial groups (Table-2). However, Puerto Rican men had a significant higher incidence $(p<0.05)$ of penile cancer in all age group categories studied (Table-2). Particularly higher incidence of penile cancer was observed in Puerto Rican men of younger ages ( $<60$ years) as compared to their USH, NHB and NHW counterparts $(p<0.05)$. Puerto Rican men younger than 60 years old had approximately 5 times higher incidence

Table 1 - Incidence and mortality for penile cancer among men in PR and racial/ethnic groups in the US: 1992-1996, 19972000, and 2001-2004.

Incidence $(x$ 100,000)

\begin{tabular}{lccccc} 
Racial/Ethnic Group & $1992-1996$ & $1997-2000$ & $2001-2004$ & $\%$ change & $\mathrm{CP} 95 \% \mathrm{Cl}$ \\
\hline PR & 3.46 & 3.19 & 2.75 & -20.59 & $-37.16,-4.02$ \\
NHW & 0.84 & 0.84 & 0.81 & -3.52 & $-18.54,11.50$ \\
NHB & 1.32 & 0.91 & 1.18 & -10.84 & $-49.04,44.47$ \\
USH & 0.94 & 1.02 & 0.92 & -2.28 & $-44.13,22.42$ \\
\hline
\end{tabular}

Mortality $(x 100,000)$

\begin{tabular}{lccccc} 
Racial/Ethnic Group & $1992-1996$ & $1997-2000$ & $2001-2004$ & $\%$ change $^{*}$ & $\mathrm{CP} 95 \% \mathrm{Cl}$ \\
\hline PR & 0.57 & 0.57 & 0.64 & 12.59 & $-40.59,65.86$ \\
NHW & 0.19 & 0.19 & 0.19 & 0.87 & $-10.02,11.74$ \\
NHB & 0.21 & 0.30 & 0.34 & 57.99 & $-45.07,0.00$ \\
USH & 0.33 & 0.27 & 0.26 & -22.56 & $-0.96,116.86$ \\
\hline
\end{tabular}

* Estimation of the percent change was done utilizing 15 decimals points. 


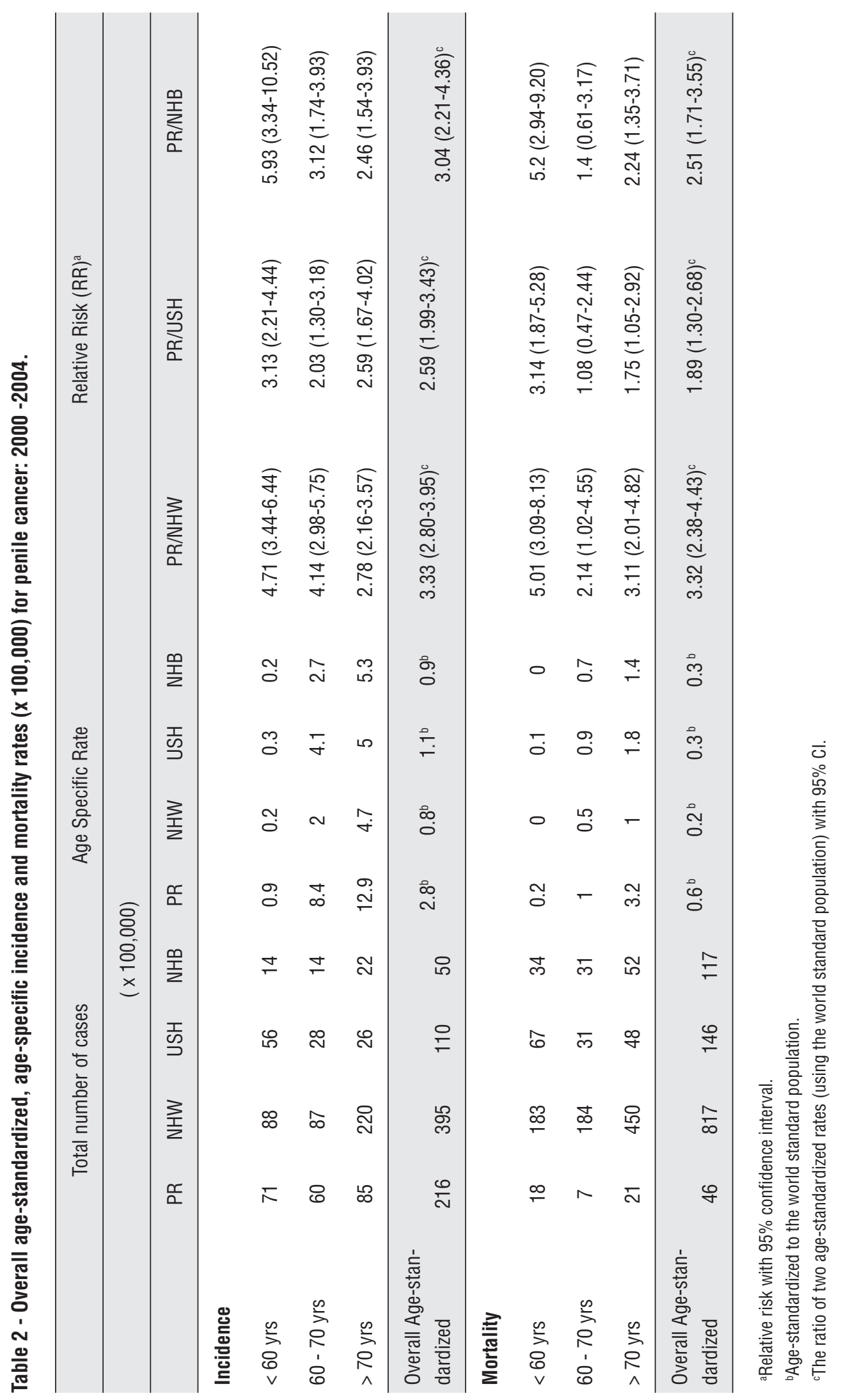


than NHW (SIR: 4.71; 95\%CI=3.44, 6.44), up to three times higher incidence than USH (SIR: $3.13 ; 95 \% \mathrm{CI}=2.21,4.44)$ and approximately 6 times higher incidence than NHB men (SIR: $5.93 ; 95 \% \mathrm{CI}=3.34,10.52$ ).

\section{Penile Cancer Mortality}

The annual mortality of penile cancer (per 100,000) ranged from 0.19 in NHW to 0.63 in PR men (Table-1). During the period 20002004, Puerto Rican men had a significant higher mortality than their USH, NHB and NHW counterparts $(p<0.05)$, with mortality up to three times higher than NHW men (SIR: 3.32; $95 \% \mathrm{CI}=2.38,4.43)$. When comparing the age-specific mortality in the same period, higher mortality were also observed among men in PR as compared to the other studied racial/ethnic groups in all age groups, except the 60-70 age group in which men in PR compared with their USH and NHB counterparts (Table-2).

\section{Socioeconomic Indicators and Penile Cancer Incidence and Mortality in PR}

As shown in Table-3, men in PR in the lowest socioeconomic deprivation index (SEP1) had 70\% higher incidence of penile cancer as compared with those PR men in the highest socioeconomic deprivation index (SEP5). However, the association was marginally significant (SRR: 1.70; 95\%CI=0.97, 2.87). When evaluating each of the eight socioeconomic indicators, only low educational attainment $(<12$ years of education), was statistically associated with higher incidence of penile cancer $(p<0.05)$. That is, men in PR with a low educational attainment had up to 2 times higher incidence of penile cancer as compared with those with high educational attainment (SRR: 2.18; 95\% CI=1.42, 3.29).

When evaluating the effect of the SEP index in penile cancer mortality, a higher mortality was also observed among those in the lowest socioeconomic deprivation index (SEP1); however, this observed increment was not statistically significant (SRR: $1.61 ; 95 \% \mathrm{CI}=0.38,5.21$ ). No statistical association was observed in the other socioeconomic indicators components ( $p>0.05$ ).

\section{DISCUSSION}

This analysis supports racial and ethnic differences in the incidence and mortality of penile cancer in the US and PR. The available data also permit the assessment of potential sub-group differences within the broad Hispanic/ Latino category and showed important variations between the incidence and mortality for PR and USH. Our study showed that although penile cancer is a relatively uncommon tumor, the incidence of this tumor is up to three times higher in PR as compared with other racial/ethnic groups in the continental US. Mortality was also significantly higher in Puerto Rican men as compared to any other racial/ethnic group.

Epidemiological studies have identified risk factors such as smoking and being uncircumcised as risk factors for penile cancer (1). Tobacco smoking, particularly current smoking, has been reported in a number of studies to be linked to increased risk of penile cancer, although other studies have failed to found support for this association (14). Smoking rates overall have been declining in the last decade (15), with lower median prevalence of adult current smoking in PR (12.2\%) as compared to the US (19.8\%), a pattern that does not support the higher incidence of penile cancer in PR as compared to the US. Although decreasing trends have been observed in population-based studies US, when differences by Hispanic origin have been examined, respondents of Puerto Rican and Cuban origin have been found to be significantly more likely to smoke (16) as compared to other Hispanic origin sub-groups.

On the other hand, low rates of circumcision in PR and USH have been reported in population-based studies. For example, the National Health and Nutrition Examination Survey (NHANES 1999-2004) reported that 79\% men in the US are circumcised (17), with prevalence estimates lower among Mexican American men (42\%). Population-based studies in PR have estimated the prevalence of circumcision among men is only $30.6 \%$ (18), highlighting a possible mechanism in which penile cancer rates might be higher in this population, although further 
Table 3 - Age-standardized $(x 100,000)$ incidence and mortality rates for Penile Cancer by SEP index: Puerto Rico 2000-2004.

\begin{tabular}{|c|c|c|c|c|}
\hline & & SEP $1^{a, b}$ & SEP5 $5^{a, c}$ & SRR $(95 \% \mathrm{Cl})^{d}$ \\
\hline \multicolumn{5}{|l|}{ Incidence } \\
\hline SEP Category & & 2.6 & 1.5 & $1.70(0.97,2.87)$ \\
\hline \multirow[t]{8}{*}{ SEP Components } & $\begin{array}{c}\text { Less than } 12 \text { years of } \\
\text { education }\end{array}$ & 3.8 & 1.7 & $2.18(1.42,3.29)$ \\
\hline & English Proficiency & 2.2 & 1.5 & $1.48(0.83,2.53)$ \\
\hline & Median Family Income & 2.5 & 1.7 & $1.50(0.87,2.46)$ \\
\hline & No Cars & 1.9 & 1.8 & $1.02(0.65,1.60)$ \\
\hline & No Telephone & 2.7 & 1.6 & $1.67(1.00,2.70)$ \\
\hline & Poverty & 2.5 & 1.7 & $1.48(0.85,2.48)$ \\
\hline & Unemployed & 2.4 & 1.6 & $1.47(0.84,2.47)$ \\
\hline & White Collar & 19.2 & 9.3 & $2.06(0.98,4.08)$ \\
\hline \multicolumn{5}{|l|}{ Mortality } \\
\hline SEP Category & & 0.5 & 0.3 & $1.61(0.38,5.21)$ \\
\hline \multirow[t]{8}{*}{ SEP Components } & $\begin{array}{c}\text { Less than } 12 \text { years of } \\
\text { Education }\end{array}$ & 2.7 & 1.8 & $1.53(0.15,9.45)$ \\
\hline & English Proficiency & 0.5 & 0.3 & $1.34(0.31,4.43)$ \\
\hline & Median Family Income & 6.2 & 2.0 & $3.14(0.63,14.68)$ \\
\hline & No Cars & 3.0 & 2.4 & $1.27(0.28,6.47)$ \\
\hline & No Telephone & 8.0 & 2.1 & $3.84(0.93,15.19)$ \\
\hline & Poverty & 0.5 & 0.4 & $1.15(0.28,3.50)$ \\
\hline & Unemployed & 3.4 & 2.0 & $1.72(0.17,10.43)$ \\
\hline & White Collar & 0.9 & 0.5 & $1.86(0.74,4.24)$ \\
\hline
\end{tabular}

a Rates are per 100,000 and age-adjusted to the $2000 \mathrm{PR}$.

b $\mathrm{SRR}=\mathrm{SEP}=1$ is the low socioeconomic position (highest socioeconomic deprivation).

${ }^{\circ} \mathrm{SEP}=5$ is the high socioeconomic position (lowest socioeconomic deprivation), reference group.

d SRR=SEP1/SEP5 indicates the standardized rate ratio with 95\% confidence intervals (Tiwari method).

e Statistically significant $(p<0.05)$ 
research is needed in this area. There is support in the literature that the effect of circumcision is mediated by avoidance of phimosis. In many countries, such as Denmark, circumcision rates have not changed but penile cancer incidence rates are falling. This may be due to better hygiene over the years with lower rates of phimosis. In $\mathrm{PR}$, determining the importance of circumcision in the incidence of penile cancer among men will be necessary to document, as there is evidence that male circumcision reduces the risk of heterosexually acquired HIV infection (19) and clearance of HPV infection, including infection with oncogenic types (20).

Past infection with HPV is also a known risk factor for penile cancer which has been detected in approximately 50\% of penile cancer cases (21). As HPV infection and lifestyle sexual partners are significantly associated with oncogenic HPV infection $(22,23)$, the number of lifetime sexual partners on penile cancer is also important to understand the role of higher number of sexual partnering and increased risk of penile cancer. In PR, a population-based study of men and women ages 18-64 reported that $80 \%$ of men initiated their sexual activity before age 18 and almost half of the men interviewed (47.8\%) had more than 7 sexual partners in their lifetime (21). Although this estimates are lower than a population-based study in the US, these results showed an early age of sexual initiation and a high prevalence of multiple sexual partners in PR, which document the importance of developing interventions that promote safe sex practices among men in this population, which will also partially protect them against HPV infection.

Clinical risk factors have also been identified as strong predictors of penile cancer. The most important clinical risk factor for invasive penile cancer is the history of phimosis (2). Studies have found that history of phimosis was reported in approximately 25\%-60\% of patients with penile cancer (24). A clinical study realized in PR reported that phimosis was present in (85.7\%) of the pathologic reports reviewed between 1979-1989 in a hospital of the western region of PR (25). Other risk factors that were explored in this study and showed significant association with penile cancer were history of Sexually Transmitted Infections (STIs), and leukopakia.

Our study also showed that the rate of penile cancer in PR has been declining. Although other studies have attributed similar declines to the availability of prophylactic HPV vaccination (7), attributing the impact of the decline of penile cancer incidence to HPV vaccination is unlikely since population uptake of the vaccine among Puerto Rico young men is very recent and low (6\%) (26) and just recently (2009) vaccination was approved for men. Other studies have attributed this decline in penile cancer incidence due to better hygiene over the years with lower rates of phimosis. Also, the impact of how other risk factors (hygiene, decrease in tobacco consumption or potential increasing circumcision rates) might have an impact on this decline cannot be ascertained utilizing this data set.

On the other hand, it is important to highlight that although a significant reduction was observed in incidence rates, an increased trend in penile cancer mortality was noted among men in PR. Although this increase in mortality was not significant, an evaluation of the clinical characteristics of cancers diagnosed with respect to extent of disease and treatment provided will be important to ascertain in future studies. Within this context, it is important to evaluate the effect of socioeconomic position on penile cancer incidence. Socioeconomic disparities in cancer have been widely reported previously (27). However, which cultural, economic or social factors might be influencing higher risk among Puerto Rican men is unknown. It can be hypothesized that health insurance coverage, access to appropriate early detection and treatment could be influencing this higher rate. Also, low educational attainment might influence the high incidence of penile cancer among PR men, due to inadequate hygiene practices, as has been highlighted (28). From this latest study the importance of education on the prevention of urogenital cancers is highlighted, as a key element to decrease the burden of penile cancer in this population.

Finally, it is important to highlight that this data showed higher incidence and mortality of penile cancer among men younger than 60 
years old, which contrasts with the reported epidemiology of penile cancer elsewhere (29). These findings highlight the opportunity to strengthen prevention efforts in Puerto Rican men. A high incidence of penile cancer and mortality within younger cohorts in Puerto Rican men might be possible due to a possible convergence of early and high sexual activity, including lifetime number of female sex partners, low circumcision rates, high prevalence of STIs and HIV in Puerto Rico, particularly among the medically underserved.

Our study has some limitations that need to be considered. Incomplete information regarding stage at diagnosis, histologic type (i.e., squamous versus melanoma versus adenocarcinoma versus urethral type), grade and sub-site of penile cancer cases in PR limits our ability to consider the impact of staging on penile cancer trends. Also, even though PR is an Hispanic population, Hispanics in the US constitute a heterogeneous group of people from a variety of Hispanic origins that show substantial variability in cancer rates. Even though the male Hispanic population residing in the US described in our study is not directly comparable to the Puerto Rican male population living in PR, racial/ethnic group comparisons identify significant disparities in the burden of penile cancer incidence and mortality and permit us generate further hypotheses about the role of environmental, genetic, social, and lifestyle factors on penile cancer occurrence.

\section{CONCLUSIONS}

In summary, this study found higher rates of penile cancer in men in PR as compared to any other studied racial/ethnic group in the US. Race/ethnic group differences can involve complex interactions between behavioral and biological processes and perhaps even social factors. The presence of this heterogeneity by race/ethnic groups needs to be acknowledged in the quantification and investigation of race/ethnic differences in penile cancer research.

\section{ACKNOWLEDGEMENTS}

The authors wish to acknowledge the collaboration of the Puerto Rico Central Cancer Registry personnel. The project described above was supported by R03 DA027939-01 and 1R03DA031590-01 from NIDA as well as U54fCA96297, U54CA96300 and NIH/NCI-U54CA096297 from the UPR/ MDACC Partnership for Excellence in Cancer Research from NCI/NIH.

\section{CONFLICT OF INTEREST}

None declared.

\section{REFERENCES}

1. Backes DM, Kurman RJ, Pimenta JM, Smith JS: Systematic review of human papillomavirus prevalence in invasive penile cancer. Cancer Causes Control. 2009; 20: 449-57.

2. Pow-Sang MR, Ferreira U, Pow-Sang JM, Nardi AC, Destefano V: Epidemiology and natural history of penile cancer. Urology. 2010; 76: S2-6.

3. Barnholtz-Sloan JS, Maldonado JL, Pow-sang J, Giuliano AR: Incidence trends in primary malignant penile cancer. Urol Oncol. 2007; 25: 361-7. Erratum in: Urol Oncol. 2008; 26: 112. Guiliano, Anna R [corrected to Giuliano, Anna R].

4. Palefsky J: HPV-Related Disease in Men: Anal and Penile Cancer, in Reducing the Global Burden of HPV-Related Disease: Cervical Cancer and Beyond. 2007. Available at: http://www.medscape.com/viewarticle/565225

5. Parkin DM, Muir CS: Cancer Incidence in Five Continents. Comparability and quality of data. IARC Sci Publ. 1992; 120: 45-173.

6. Wabinga HR, Parkin DM, Wabwire-Mangen F, Nambooze S: Trends in cancer incidence in Kyadondo County, Uganda, 1960-1997. Br J Cancer. 2000; 82: 1585-92.

7. Hernandez BY, Barnholtz-Sloan J, German RR, Giuliano A, Goodman MT, King JB, et al.: Burden of invasive squamous cell carcinoma of the penis in the United States, 19982003. Cancer. 2008 Nov 15; 113(10 Suppl): 2883-91.

8. Ortiz AP, Soto-Salgado M, Calo WA, Tortolero-Luna G, Pérez CM, Romero CJ, et al.: Incidence and mortality rates of selected infection-related cancers in Puerto Rico and in the United States. Infect Agent Cancer. 2010; 5: 10.

9. Wideroff L, Schottenfeld D: Penile Cancer. In Cancer Epidemiology and Prevention. 3rd edition. Edited by Schottenfeld $D$ and Fraumeni JF. New York: Oxford University Press; 2006: pp. 166-171.

10. Suárez E, Calo WA, Hernández EY, Diaz EC, Figueroa NR, Ortiz AP: Age-standardized incidence and mortality rates of oral and pharyngeal cancer in Puerto Rico and among NonHispanics Whites, Non-Hispanic Blacks, and Hispanics in the USA. BMC Cancer. 2009; 9: 129. 
11. Colón-López V, Ortiz AP, Palefsky J: Burden of human papillomavirus infection and related comorbidities in men: implications for research, disease prevention and health promotion among Hispanic men. P R Health Sci J. 2010; 29: 232-40.

12. Surveillance Epidemiology and End Results. Policy for calculating Hispanic Mortality. National Cancer Institute, National Institutes of Health, U.S. Department of Health and Human Services. Bethesda. Available at. http://seer.cancer. gov/data/metadata.html

13. Torres-Cintrón M, Ortiz AP, Ortiz-Ortiz KJ, et al. Measuring Health Disparities in Cancer: The Puerto Rico Case. Submitted in Preventing Chronic Disease.

14. Brinton LA, Li JY, Rong SD, Huang S, Xiao BS, Shi BG, et al.: Risk factors for penile cancer: results from a casecontrol study in China. Int J Cancer. 1991; 47: 504-9.

15. Centers for Disease Control and Prevention (CDC): Statespecific prevalence and trends in adult cigarette smoking-United States, 1998-2007. MMWR Morb Mortal Wkly Rep. 2009; 58: 221-6.

16. Pérez-Stable EJ, Ramirez A, Villareal R, Talavera GA, Trapido E, Suarez L, et al.: Cigarette smoking behavior among US Latino men and women from different countries of origin. Am J Public Health. 2001; 91: 1424-30.

17. Xu F, Markowitz LE, Sternberg MR, Aral SO: Prevalence of circumcision and herpes simplex virus type 2 infection in men in the United States: the National Health and Nutrition Examination Survey (NHANES), 1999-2004. Sex Transm Dis. 2007; 34: 479-84.

18. Ortiz AP, Soto-Salgado M, Suárez E, del Carmen SantosOrtiz M, Tortolero-Luna G, Pérez CM: Sexual behaviors among adults in Puerto Rico: a population-based study. J Sex Med. 2011; 8: 2439-49.

19. Bailey RC, Moses S, Parker CB, Agot K, Maclean I, Krieger JN, et al.: Male circumcision for HIV prevention in young men in Kisumu, Kenya: a randomised controlled trial. Lancet. 2007; 369: 643-56.

20. Hernandez BY, Shvetsov YB, Goodman MT, Wilkens LR, Thompson $\mathrm{P}$, Zhu X, et al.: Reduced clearance of penile human papillomavirus infection in uncircumcised men. $J$ Infect Dis. 2010; 201: 1340-3.

21. Grulich AE, Jin F, Conway EL, Stein AN, Hocking J: Cancers attributable to human papillomavirus infection. Sex Health. 2010; 7: 244-52.

22. Almonte M, Albero G, Molano M, Carcamo C, García PJ, Pérez G: Risk factors for human papillomavirus exposure and co-factors for cervical cancer in Latin America and the Caribbean. Vaccine. 2008; 26(Suppl 11): L16-36

23. Vardas E, Giuliano AR, Goldstone S, Palefsky JM, Moreira ED Jr, Penny ME, et al.: External genital human papillomavirus prevalence and associated factors among heterosexual men on 5 continents. J Infect Dis. 2011; 203: 58-65.

24. Favorito LA, Nardi AC, Ronalsa M, Zequi SC, Sampaio FJ, Glina S: Epidemiologic study on penile cancer in Brazil. Int Braz J Urol. 2008; 34: 587-91; discussion 591-3.

25. Hunter-Mellado R, Rodríguez P: Squamous cell carcinoma of the penis. Bol Asoc Med P R. 1990; 82: 416-8.

26. Personal Communication: PR Department of Health. Vaccination Program. June 20; 2012.

27. Krieger N, Williams DR, Moss NE: Measuring social class in US public health research: concepts, methodologies, and guidelines. Annu Rev Public Health. 1997; 18: 341-78.

28. de Souza KW, dos Reis PE, Gomes IP, de Carvalho EC: Prevention strategies for testicular and penile cancer: an integrative review. Rev Esc Enferm USP. 2011; 45: 277-82.

29. Mosconi AM, Roila F, Gatta G, Theodore C: Cancer of the penis. Crit Rev Oncol Hematol. 2005; 53: 165-77.

Correspondence address:

Dr. Vivian Colón-López Associate Professor

University of Puerto Rico, Medical Sciences Campus, Graduate School of Public Health PO BOX 365067

San Juan, 00936-5067, Puerto Rico Fax: +7 87 757-0772 E-mail: vivian.colon@upr.edu 


\section{EDITORIAL COMIMENT}

This is a well done epidemiologic comparison among PR and American men regarding penile cancer. As expected, low educational level is a determinant for higher incidence and mortality due this malignancy. Penile cancer is a health concern in poor regions worldwide. The findings of this study are result of an elegant and complex statistical job. Unhapily it was not possible to control by stage the mortality analysis, because tumor stage information were lacking in this large retrospective data base.

Although the authors became impressed due the higher incidence and mortality rates in men younger than 60 years old, this situation is not so uncommon in developing countries. In a national survey, sponsored by Brazilian Urological Society, Favorito et al. showed, almost 20\% of penile cancer affecting men under 45 years old, being, 3.53\% under 26 years and 3.88\% under 35 years and 12\%, under 45 years old (1), reinforcing the idea that penile cancer is a mutilant disease, which can affect men in the most productive phase of their lives. As the authors stressed in the discussion issues about behavioral aspects and lifestyle characteristics as risk factors for penile cancer, in future studies in PR populations, questions about penile traumas (2), [references 2 and 17] and about sex with animals (3), might be added.

\section{REFERENCES}

1. Favorito LA, Nardi AC, Ronalsa M, Zequi SC, Sampaio FJ, Glina S: Epidemiologic study on penile cancer in Brazil. Int Braz J Urol. 2008; 34: 587-91; discussion 591-3.

2. Tsen HF, Morgenstern H, Mack T, Peters RK: Risk factors for penile cancer: results of a population-based case-control study in Los Angeles County (United States). Cancer Causes Control. 2001; 12: 267-77.
3. Zequi Sde C, Guimarães GC, da Fonseca FP, Ferreira U, de Matheus WE, Reis LO, et al.: Sex with animals (SWA): behavioral characteristics and possible association with penile cancer. A multicenter study. J Sex Med. 2012; 9: 1860-7.

Dr. Stênio de Cássio Zequi Urologia / Oncologia, Fundação Antônio Prudente - São Paulo Rua Batataes, 391 - 4o. andar, Jardim Paulista São Paulo, SP, 01423-010, Brazil E-mail:steniozequi@uol.com.br 